\title{
The Control of Brushless DC Motor for Electric Vehicle by Using Chaotic Synchronization Method
}

\author{
Ercan KÖSE ${ }^{1 *}$, Aydın MÜHÜRC $\ddot{U ̈}^{2}$ \\ ${ }^{1}$ Tarsus University, Takbaş Mevkii, Tarsus-Mersin, 33480, Turkey \\ ercankos@gmail.com (*Correspondingauthor) \\ ${ }^{2}$ Kırklareli University, Kayalı Yerleşkesi-Kırklareli, Turkey \\ amuhurcu@klu.edu.tr
}

\begin{abstract}
Brushless DC motor (BLDC) used in electric vehicles can be operated in normal weather conditions and on straight roads, with chaotic dynamics of BLDC motor and high efficiency at a certain stable point. The dynamics of the BLDC motor applied on the electric car can be constantly changed according to the road conditions and wind speed. In this study, chaotic synchronization methods were developed to convert the BLDC motor into the desired reference chaotic dynamics. Synchronization method is developed based on sliding mode control (SMC), PI control and adaptive control methods. The artificial bee colony algorithm has been used to calculate the optimal values of the Kp and Ki coefficients of the PI controller. The numerical simulation results showing the performances of the controllers were obtained in MATLABSimulink environment. In order to better compare the performance of the controllers, the error performance indices have been provided according to these three control methods. All the three methods have shown that the chaotic based BLDC motor can be controlled, but sliding mode control method has proved to be a better performer.
\end{abstract}

Keywords: Brushless DC motor (BLDC), Chaotic dynamics, Synchronization, Sliding mode control (SMC), PI control.

\section{Introduction}

In everyday life, chaos described as disorder and irregularity, has regularity in itself. These systems exhibiting complex dynamic behaviors have been modelled and explained using chaotic systems. These systems have found significant application areas in science and engineering since their development by Lorenz to model weather events in 1963 (Lorenz, 1963). Some important ones among these application areas are cryptography; secure communication, biomedical, robotics.

As a result of the intensive use of Internet in recent years, the need for secure communication with the encryption of information has arisen as the trade has moved to electronic media. Nowadays, credit cards and other e-commerce information can be easily stolen and used in digital environment. Also, mobile phone conversations can be tapped. Digital information theft has forced banks and other institutions to take new precautions on infrastructure for secure communication. New computer programs emerging in this direction use encryption methods for data communication. Chaotic systems have been widely used for data encryption due to their nonlinear complex behaviors, being sensitive to the initial conditions and it is highly difficult to predict.

Scientists have proposed many chaotic models and new methods based on these models for encryption. Synchronization is one of the most important building blocks for data encryption.
"Synchronous" has the meaning of occurring at the same time or being in the same moment. Besides, synchronization means harmonization in terms of phase and frequency between two changing signals. Pecora and Carroll showed in 1990 that the chaotic systems could be synchronized by connecting them to common signals in the study of synchronization in systems, with examples of Lorenz and Rösler Chaotic Systems (Pecora \& Caroll, 1990). There have been many studies on synchronization from 1990 until the present day. Some of the important studies on this topic over the past few years are as follows: Vaidyanathan and Azar performed adaptive synchronization of the unknown system parameters with the novel chaotic system and supported the results with Lyapunov stability theory (Vaidyanathan \& Azar, 2015). In another study, Khan et al. performed the synchronization of the Genesio and Lü chaotic systems by using the active control method (Khan et al., 2016). In a different study, Hou designed a novel hyper with the evolutionary programming (EP)-based PID controller, and experimentally proved the validity of the synchronization he proposed (Hou, 2017).

Chaotic systems can be used to analyse and control many dynamic structures. One of these structures is represented by the BLDC motors. Brushless DC motors have found widespread use especially in electric vehicles and electric bicycles, which have been on the agenda in recent years. 
Different types of controller approaches have been needed so that these motors can be operated at the desired stability and at high efficiency points. In line with these views, Ibrahim et al. performed speed control of BLDC motor in Matlab/Simulink environment using PID controller based on particle swarm optimization (BF), and evaluated the efficiency of the proposed method according to the steady-state error, rise time, settling time and maximum overshoot, performance criteria (Ibrahim et al., 2014). Some important studies performed for the BLDC engine are as follows. Varshney et al. investigated the speed response of a BLDC motor in a simulation environment using a fuzzy PID controller under-sudden and gradual change load condition (Varshney et al., 2017). Also, Rajagopal et al. made investigations about the dynamic properties of the fractional order BLDC motor model, and performed its chaos control of the fractional order (Rajagopal et al., 2017).

In recent years, measures have been taken to reduce the emission values of vehicles with internal combustion engines that cause greenhouse gases. Although internal combustion engine volumes have been reduced recently, a large number of studies have also been carried out to make zero emission electric vehicles more efficient. Most electric vehicles use BLDC motors. The energy consumption and performance of BLDC motors vary under different operating conditions. The better performance would mean increased vehicle range and lower energy consumption. In the literature, there are very few studies related to the control of chaotic models of BLDC motors. In addition, the synchronization method is often used for communication and encryption purposes. Therefore, this investigation will serve as a good example for the use of synchronization as a control method. This study will also provide a novel approach to the issues regarding to the control of chaotic models of BLDC motors in the literature.In the current introduction, the definition of chaos, the importance of cryptography, the synchronization in chaotic systems, the important studies in the related literature and finally the control of BLDC motors have been presented. The chaotic structure of the BLDC motors is discussed mainly in Section 2, whereas section 3 is focused on the control design synchronized with the SMC method. Section 4, however, presents the stability analysis and the PI controller structure, whilst the adaptive synchronization control of the BLDC is discussed in section 5. The numerical simulation results are also given in Section 6. Finally, Section 7 is structured based on the overall results obtained from the investigation and draws a conclusion.

\section{The Model of BLDC Motor}

The chaotic model of the BLDC motor used as the master system in the synchronization method can be given as follows according to the references (Hemati \& Leu, 1992; Premkumar \& Manikandan, 2015; Rajagopal et al., 2017).

The master system:

$$
\left.\begin{array}{l}
\dot{x}_{m}=v_{q}-x_{m}-y_{m} z_{m}+\rho z_{m} \\
\dot{y}_{m}=v_{d}-\delta y_{m}+x_{m} z_{m} \\
\dot{z}_{m}=\sigma\left(x_{m}-z_{m}\right)+\eta x_{m} y_{m}-T_{L}
\end{array}\right\},
$$

The slave systems for control input $\left(u_{i}\right)$ can be expressed as follows:

$$
\left.\begin{array}{l}
\dot{x}_{s}=\tilde{v}_{q}-x_{s}-y_{s} z_{s}+\tilde{\rho} z_{s}+u_{x} \\
\dot{y}_{s}=\tilde{v}_{d}-\tilde{\delta} y_{s}+x_{s} z_{s}+u_{y} \\
\dot{z}_{s}=\tilde{\sigma}\left(x_{s}-z_{s}\right)+\tilde{\eta} x_{s} y_{s}-\tilde{T}_{L}+u_{z}
\end{array}\right\},
$$

The unknown parameters of the slave system are defined as $\tilde{v}_{q}, \tilde{\rho}, \tilde{v}_{d}, \tilde{\delta}, \tilde{\sigma}, \tilde{\eta}, \tilde{T}_{L}$.

The synchronization errors are required to be zero for full synchronization of master and slave systems. In this case, the synchronization error can be shown mathematically as the $\lim _{t \rightarrow \infty} e_{i}=0$ where $i=x, y, z$ and $e_{i}$ is described as $e_{i}=i_{m}-i_{s}$.'

The error dynamics can be defined as, $\tilde{e}_{i}=\tilde{i}_{m}-\tilde{i}_{s}$.

According to the error dynamics described above, the error dynamics structure of master and slave systems can be defined as follows:

$$
\left.\begin{array}{rl}
\dot{e}_{x}= & v_{q}-\tilde{v}_{q}-\left(x_{m}-x_{s}\right)-\left(y_{m} z_{m}-y_{s} z_{s}\right) \\
& +\rho z_{m}-\tilde{\rho} z_{m}-u_{x} \\
\dot{e}_{y}= & v_{d}-\tilde{v}_{d}-\left(\delta y_{m}-\tilde{\delta} y_{s}\right)+x_{m} z_{m}-x_{s} z_{s}-u_{y} \\
\dot{e}_{z}= & \sigma\left(x_{m}-z_{m}\right)-\tilde{\sigma}\left(x_{s}-z_{s}\right)+\eta x_{m} y_{m} \\
& -\tilde{\eta} x_{s} y_{s}-\left(T_{L}-\tilde{T}_{L}\right)-u_{z}
\end{array}\right\},
$$




\section{The Synchronization Controller Design Using SMC Method}

The SMC has a robust control algorithm against dynamic uncertainty. The SMC is performed in two steps as a sliding surface and as a switching control law. For the sliding phase in the SCM have been chosen the integral sliding surface. The integral sliding surface was described by Rajagopal, K. et al in 2017 (Rajagopal et al., 2017) as follows: $s_{i}=e_{i}+k_{i} \int e_{i}(\tau) d \tau$, where $i=x, y, z \quad k_{i}>0$.

The time derivative of the sliding surface can be shown as $\dot{s}_{i}=\dot{e}_{i}+k_{i} e_{i}$ If the $s=0$ and $\dot{s}_{i}=0$, the system is in the sliding-mode phase.

The parameter estimation errors are given by: $e_{v_{q}}=v_{q}-\tilde{v}_{q}, e_{p}=p-\tilde{p}, e_{v_{d}}=v_{d}-\tilde{v}_{d}, e_{\delta}=\delta-\tilde{\delta}$, $e_{\sigma}=\sigma-\tilde{\sigma}, e_{\eta}=\eta-\tilde{\eta}$ and $e_{T_{L}}=T_{L}-\tilde{T}_{L}$.

The SMC can be expressed as follows:

$$
\left.\begin{array}{l}
e_{x}=x_{m}-x_{s} \\
e_{y}=y_{m}-y_{s} \\
e_{z}=z_{m}-z_{s}
\end{array}\right\}
$$

The $e_{x}(t), e_{y}(t)$ and $e_{z}(t)$ in the Equation (4) have been show synchronization errors.

$$
\left.\begin{array}{l}
\dot{e}_{x}=e_{v_{s}}-e_{x}-\left(y_{m} z_{m}-y_{s} z_{s}\right)+p z_{m}-\tilde{p}_{s}-u_{x} \\
\dot{e}_{y}=e_{v_{d}}+x_{m} z_{m}-x_{s} z_{s}-\left(\delta y_{m}-\tilde{\delta} y_{s}\right)-u_{y} \\
\dot{e}_{z}=\sigma\left(x_{m}-z_{m}\right)-\tilde{\sigma}\left(x_{s}-z_{s}\right)+\eta x_{m} y_{m}-\tilde{\eta} x_{s} y_{s}-e_{T_{L}}-u_{z}
\end{array}\right\},
$$

By the definitions of the reaching law (Köse, 2017), $\dot{s}_{i}=-\gamma_{i} \operatorname{sgn}\left(s_{i}\right)-k_{i} s_{i}, i=x, y, z$ and $\gamma_{i}>0$ and $k_{i}>0$ are the sliding surface gains. If the access surface is selected as this,

$$
\left.\begin{array}{c}
\dot{s}_{x}=-\gamma_{x} \operatorname{sgn}\left(s_{x}\right)-k_{x} s_{x} \\
\dot{s}_{y}=-\gamma_{y} \operatorname{sgn}\left(s_{y}\right)-k_{y} s_{y} \\
\dot{s}_{z}=-\gamma_{z} \operatorname{sgn}\left(s_{z}\right)-k_{z} s_{z}
\end{array}\right\},
$$

According to integral sliding mode control (ISMC), $\dot{s}_{i}=\dot{e}_{i}+k_{i} e_{i}$ can be expressed as.

$$
\left.\begin{array}{c}
\dot{s}_{x}=\dot{e}_{x}+\lambda_{x} e_{x}=-\gamma_{x} \operatorname{sgn}\left(s_{x}\right)-k_{x} s_{x} \\
\dot{s}_{y}=\dot{e}_{y}+\lambda_{y} e_{y}=-\gamma_{y} \operatorname{sgn}\left(s_{y}\right)-k_{y} s_{y} \\
\dot{s}_{z}=\dot{e}_{z}+\lambda_{z} e_{z}=-\gamma_{z} \operatorname{sgn}\left(s_{z}\right)-k_{z} s_{z}
\end{array}\right\},
$$

As controller inputs $u_{x}, u_{y}$ and $u_{z}$ can be defined as below equality (8).

$$
\left.\begin{array}{rl}
u_{x}= & e_{v_{q}}-e_{x}+\left(y_{s} z_{s}-y_{m} z_{m}\right)+\rho z_{m}- \\
& \tilde{p} z_{s}+\lambda_{x} e_{x}+\gamma_{x} \operatorname{sgn}\left(s_{x}\right)+k_{x} s_{x} \\
u_{y}= & e_{v_{d}}+x_{m} z_{m}-x_{s} z_{s}-\left(\delta y_{m}-\tilde{\delta} y_{s}\right)+ \\
& \lambda_{y} e_{y}+\gamma_{y} \operatorname{sgn}\left(s_{y}\right)+k_{y} s_{y} \\
u_{z}= & \sigma\left(x_{m}-z_{m}\right)-\tilde{\sigma}\left(x_{s}-z_{s}\right)+\eta x_{m} y_{m}- \\
& \tilde{\eta} x_{s} y_{s}-e_{T_{L}}+\lambda_{z} e_{z}+\gamma_{z} \operatorname{sgn}\left(s_{z}\right)+k_{z} s_{z}
\end{array}\right\}
$$

Constants $\lambda_{x}, \lambda_{y}, \lambda_{z}, \gamma_{x}, \gamma_{y}$ and $\gamma_{z}$ given in Equation (8) are positive integers.

\section{The Stability Analysis of Synchronization Controller}

The results are confirmed using Lyapunov stability theory (Khalil, 2001). For stability control, the following quadratic Lyapunov function is used:

$V\left(s_{x}, s_{y}, s_{z}\right)=\frac{1}{2}\left(s_{x}^{2}+s_{y}^{2}+s_{z}^{2}\right)$

The time-derivative of $\mathrm{V}$ is calculated as,

$$
\left.\begin{array}{rl}
\dot{V} & =s_{x} \dot{s}_{x}+s_{y} \dot{s}_{y}+s_{z} \dot{s}_{z}, \\
\dot{V}= & s_{x}\left(-\gamma_{x} \operatorname{sgn}\left(s_{x}\right)-k_{x} s_{x}\right)+s_{y}\left(-\gamma_{y} \operatorname{sgn}\left(s_{y}\right)\right. \\
& \left.-k_{y} s_{y}\right)+s_{z}\left(-\gamma_{z} \operatorname{sgn}\left(s_{z}\right)-k_{z} s_{z}\right)
\end{array}\right\},
$$

Simplifying $\dot{V}$ equation, it is obtained:

$\dot{V}=-\gamma_{x}\left|s_{x}\right|-k_{x} s_{x}^{2}-\gamma_{y}\left|s_{y}\right|-k_{y} s_{y}^{2}-\gamma_{z}\left|s_{z}\right|-k_{z} s_{z}^{2}$,

$\gamma_{x}, \gamma_{y}, \gamma_{z}, k_{x}, k_{y}$ and $k_{z}$ are positive constants, but $\dot{V}$ is a negative definite function.

The BLDC master and slave system have exhibited chaotic oscillations using the initial condition values. The starting conditions of the master system have been used in Reference 14. The values for the master system parameters are: $v_{q}=0.168$, $\rho=60, v_{d}=20.66, \delta=0.875, \eta=0.26, T_{L}=0.53$ and $\sigma=4.55$. The master system state variables of the initial condition values are: $x(0)=3.63$, $y(0)=56.02, z(0)=0.29$. The values for the slave system parameters $v_{q}=0.160, \rho=58, v_{d}=21$, $\delta=0.8, \eta=0.23, T_{L}=0.52$ and $\sigma=4.40$ have been chosen. The slave system state variables of the initial condition values $x(0)=3.65, y(0)=56$ 
and $z(0)=0.35$ have been chosen. The designed $\mathrm{SMC}$ control structure is shown in Fig. 1.

Other study proposes a proportional-integral (PI) control structure as it is shown in Fig. 2.

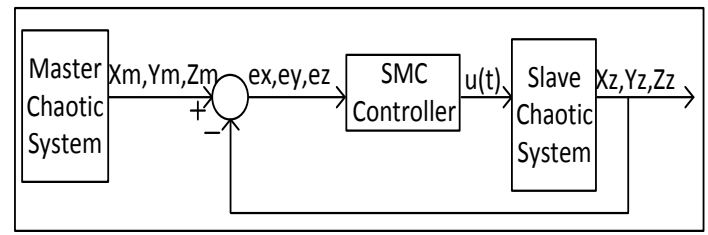

Figure 1. Block diagram of chaotic synchronization using SMC control method

The PID control method is a reference control method that has been used since 1940s and is widely spread in practical applications. The PI control scheme given in Fig. 2 is used in Reference 7 and is preferred because it performs well in nonlinear chaotic system control. The artificial bee colony algorithm was developed being inspired by the honey bees' intelligent behaviour in honey (Karaboga \& Basturk, 2007).

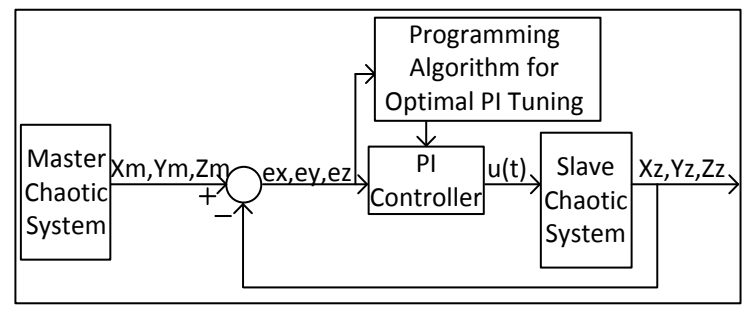

Figure 2. Block diagram of chaotic synchronization using PI control method

This algorithm, which is heuristic, have given very good answers in optimization problems. This control system has been designed by using heuristic artificial bee colony programming algorithm for optimal PI tuning. In this system, control error indices as IAE (integral of the absolute error), ISE (integral of square error), ITAE (the integral of the time multiplied by the absolute value of the error) and ISCI (the integral of the system responses have been examined. The slave system in Fig. 5(b) is identical to the master system except for the initial conditions $z(0)=0.23$ The initial conditions and constants of the slave system in Fig. 5(c), as given above are all different from the master system. The convergence curves for these error indices used for optimization are given in Fig. 3. Optimal and values for the PI controller coefficients are shown in Table 1.
Table 1. Optimal Kp and Ki values for the PI controller

\begin{tabular}{|c|c|c|}
\hline $\begin{array}{c}\text { State } \\
\text { Variables }\end{array}$ & Kp & Ki \\
\hline $\mathrm{x}$ & 44.6056 & 11.6178 \\
\hline $\mathrm{y}$ & 48.9657 & 46.7987 \\
\hline $\mathrm{z}$ & 50.3248 & 39.0856 \\
\hline
\end{tabular}

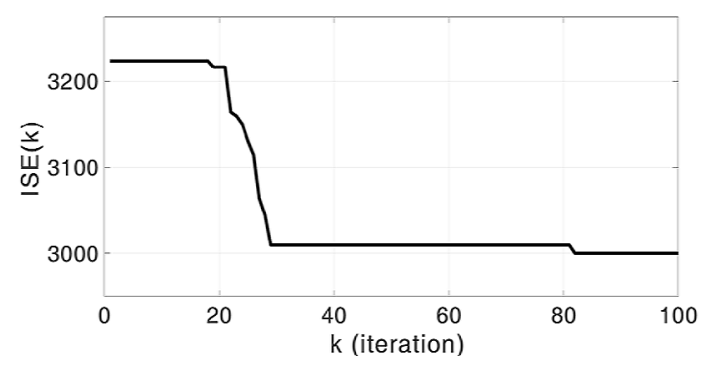

Figure 3. Converge curves of ISE (integral of square)

\section{Adaptive Synchronization Control of The BLDC Motor}

The adaptive control is a control method that can adjust itself to the changes of the system depending on the parameter estimated. In addition, Lyapunov stability theory can be used to control the stability of systems based on modified estimates. The adaptive control design for this system has been performed step by step as indicated below.

The master and slave system Equation (1) and (2) of the BLDC motor to be controlled are defined. Equation (3) was obtained by using these equality definitions. Equation (3) as depending on error dynamics can be expressed as in Equation (13). The error dynamics,

$\left.\begin{array}{l}\dot{e}_{x}=-e_{x}-e_{y} e_{z}-z_{m} e_{y}-x_{m} e_{z}-p e_{z}+u_{x} \\ \dot{e}_{y}=-\delta e_{y}+e_{x} e_{z}+x_{m} e_{z}+z_{m} e_{x}+u_{y} \\ \dot{e}_{z}=\sigma\left(e_{x}-e_{z}\right)+\eta\left(e_{x} e_{y}+y_{m} e_{x}+x_{m} e_{y}\right)+u_{z}\end{array}\right\}$,

where $u_{x}, u_{y}, u_{z}$ are adaptive controllers to be designed using the states $x, y, z$.

$\left.\begin{array}{l}u_{x}=e_{x}+e_{y} e_{z}+z_{m} e_{y}+x_{m} e_{z}+\rho e_{z}-k_{x} e_{x} \\ u_{y}=\delta e_{y}-e_{x} e_{z}-x_{m} e_{z}-z_{m} e_{x}-k_{y} e_{y} \\ u_{z}=-\sigma\left(e_{x}-e_{z}\right)-\eta\left(e_{x} e_{y}+y_{m} e_{x}+x_{m} e_{y}\right)-k_{z} e_{z}\end{array}\right\}$,

Using the equations (13) and (14), the following closed loop error dynamics can be obtained. 
$\dot{e}_{x}=(\tilde{\rho}-p) e_{z}-k_{x} e_{x}$

$\dot{e}_{y}=(\tilde{\delta}-\delta) e_{y}-k_{y} e_{y}$

$\left.\dot{e}_{z}=(\tilde{\sigma}-\sigma)\left(e_{x}-e_{z}\right)+(\tilde{\eta}-\eta)\left(e_{x} e_{y}+y_{m} e_{x}+x_{m} e_{y}\right)-k_{z} e_{z}\right)$

The parameter estimation errors are described as follows:

$e_{p}=p-\tilde{p}, e_{\delta}=\delta-\tilde{\delta}, e_{\sigma}=\sigma-\tilde{\sigma}$ and $e_{\eta}=\eta-\tilde{\eta}$.

If the system synchronization errors are reorganized,

$$
\left.\begin{array}{l}
\dot{e}_{x}=e_{\rho} e_{z}-k_{x} e_{x} \\
\dot{e}_{y}=e_{\delta} e_{y}-k_{y} e_{y} \\
\dot{e}_{z}=e_{\sigma}\left(e_{x}-e_{z}\right)+e_{\eta}\left(e_{x} e_{y}+y_{m} e_{x}+x_{m} e_{y}\right)-k_{z} e_{z}
\end{array}\right\},
$$

The derivative of parameter estimation errors is received,

$$
\left.\left.\begin{array}{r}
e_{\rho}=\rho-\tilde{\rho}(t) \\
e_{\delta}=\delta-\tilde{\delta}(t) \\
e_{\sigma}=\sigma-\tilde{\sigma}(t) \\
e_{\eta}=\eta-\tilde{\eta}(t)
\end{array}\right\} \Rightarrow \begin{array}{r}
\dot{e}_{\rho}=-\dot{\tilde{\rho}}(t) \\
\dot{e}_{\delta}=-\dot{\tilde{\delta}}(t) \\
\dot{e}_{\sigma}=-\dot{\tilde{\sigma}}(t) \\
\dot{e}_{\eta}=-\dot{\tilde{\eta}}(t)
\end{array}\right\},
$$

and the Lyapunov approach is applied.

$$
\left.\begin{array}{l}
V\left(e_{x}, e_{y}, e_{z}, e_{\rho}, e_{\delta}, e_{\sigma}, e_{\eta}\right)=\frac{1}{2}\left(e_{x}^{2}+e_{y}^{2}\right. \\
\left.+e_{z}^{2}+e_{\rho}^{2}+e_{\delta}^{2}+e_{\sigma}^{2}+e_{\eta}^{2}\right)
\end{array}\right\},
$$

If the derivative of the above system is derived, the following equation is obtained.

$$
\left.\begin{array}{rl}
\dot{V}= & \left(e_{x} \dot{e}_{x}+e_{y} \dot{e}_{y}+e_{z} \dot{e}_{z}+e_{\rho} \dot{e}_{\rho}\right. \\
& \left.+e_{\delta} \dot{e}_{\delta}+e_{\sigma} \dot{e}_{\sigma}+e_{\eta} \dot{e}_{\eta}\right)
\end{array}\right\}
$$

Then,

$$
\left.\begin{array}{rl}
\dot{V}= & -k_{x} e_{x}^{2}-k_{y} e_{y}^{2}-k_{z} e_{z}^{2}+e_{\rho}\left(e_{x} e_{z}-\dot{\tilde{\rho}}\right) \\
& +e_{\delta}\left(e_{y}^{2}-\dot{\tilde{\delta}}\right)+e_{\sigma}\left(e_{x} e_{z}-e_{z}^{2}-\dot{\tilde{\sigma}}\right) \\
& +e_{\eta}\left(e_{z}\left(e_{x} e_{y}+\mathrm{y}_{m} e_{x}+x_{m} e_{y}\right)-\dot{\tilde{\eta}}\right)
\end{array}\right\},
$$

According to Eq. (20), the parameters are defined as follows:

$$
\left.\begin{array}{l}
\dot{\tilde{\rho}}(t)=e_{x} e_{z} \\
\dot{\tilde{\delta}}(t)=e_{y}^{2} \\
\dot{\tilde{\sigma}}(t)=e_{x} e_{z}-e_{z}^{2} \\
\dot{\tilde{\eta}}(t)=e_{z}\left(e_{x} e_{y}+y_{m} e_{x}+x_{m} e_{y}\right)
\end{array}\right\},
$$

The starting conditions of the master and slave systems have been used in the Reference 14 for numerical results. The numerical results applied using the above mathematical definitions are given in Section 6. In addition, the designed adaptive control structure is given in Fig. 4.

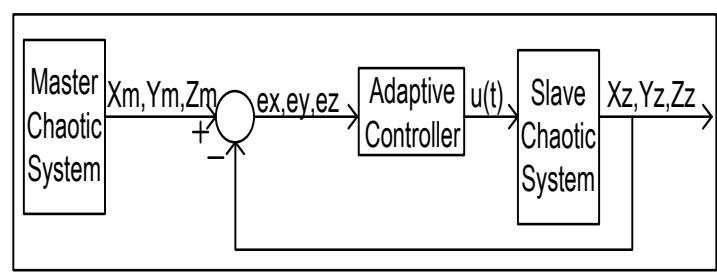

Figure 4. Block diagram of chaotic system using adaptive control method

\section{Simulation Results}

The closed loop synchronization control structures designed and developed in sections 3, 4 and 5 were used for simulation results. The simulation results of the BLDC motor are shown in Figures 5, 6, 7, $8,9,10,11,12$, and 13 depending on the starting conditions given above. The simulated results obtained in Fig.5 and Fig.6 are no controlled or open loop system responses. The results obtained in Figures 5 (a), 5 (b) and 5 (c) clearly show that a very small change in initial conditions can change the chaotic behavior too much. In addition, the simulation results have shown the performances of the controllers on the system.

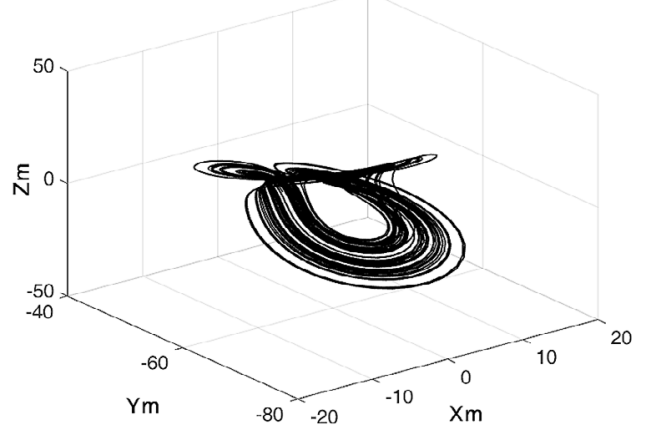

(a)

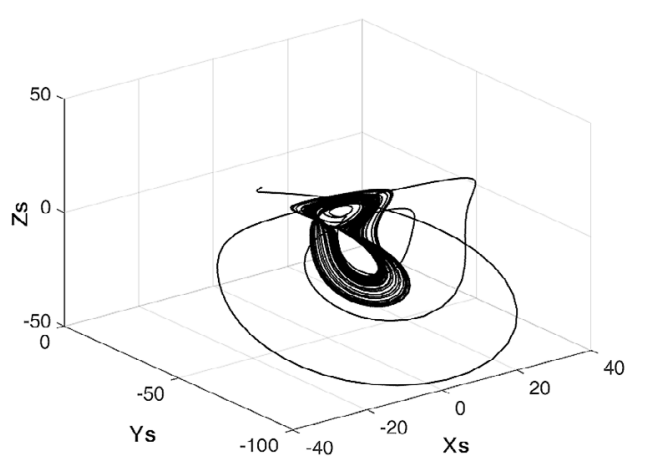

(b) 


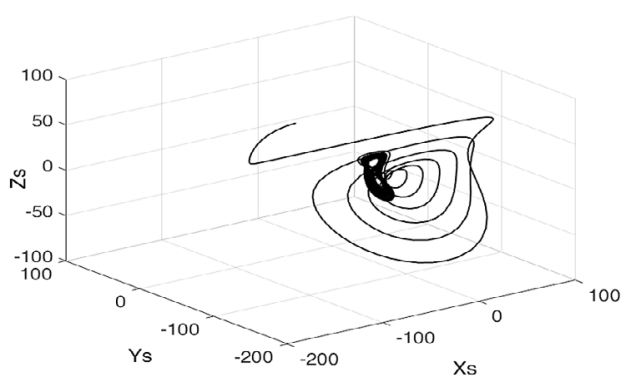

(c)

Figure 5. The 3D state portrait for master (a) and slave (b-c) chaotic system models of BLDC motor

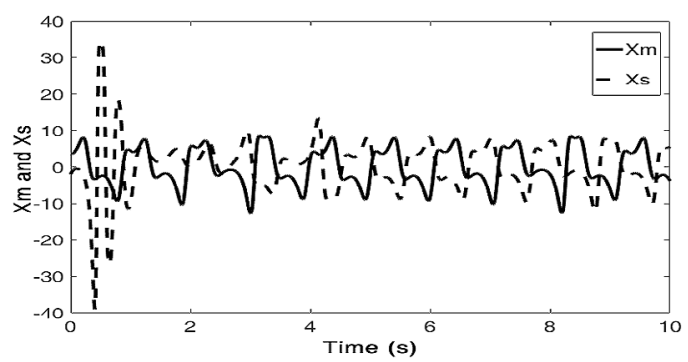

(a)

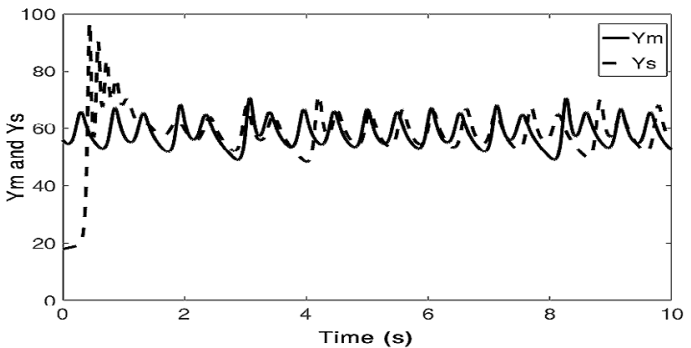

(b)

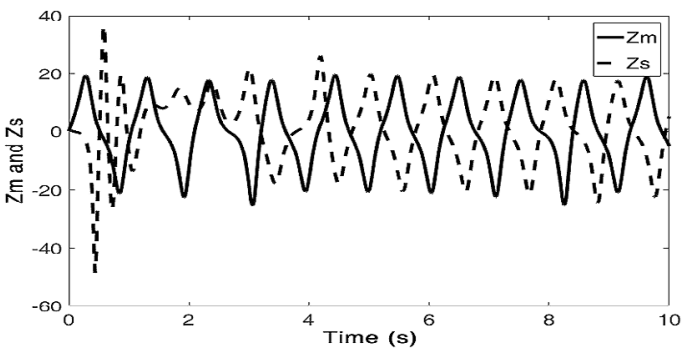

(c)

Figure 6. The time domain waveform of the $x$, $y$ and $z$ 1D state variables for master and slave chaotic systems

The sliding mode controller method described in Section 3 was applied to the closed loop control structure based on Fig. 1, and the obtained simulation results are shown in Figures 7, 8, and 9. The switching control changes for $u_{x}, u_{y}$ and $u_{z}$ controller outputs are given in Fig. 7.

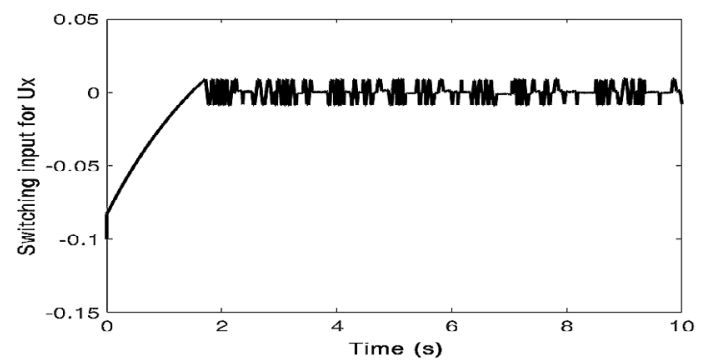

(a)

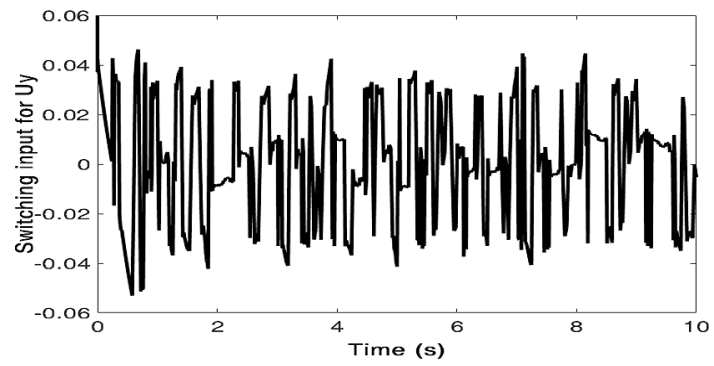

(b)

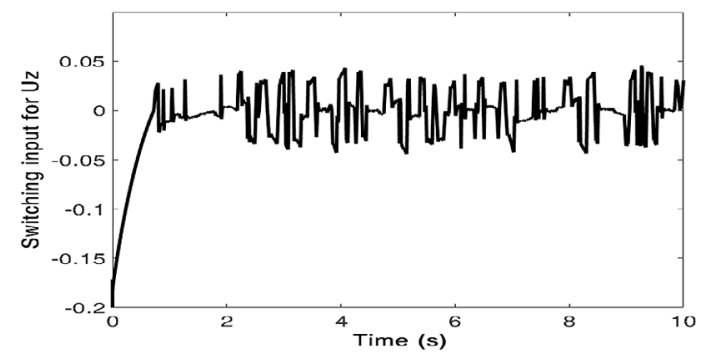

(c)

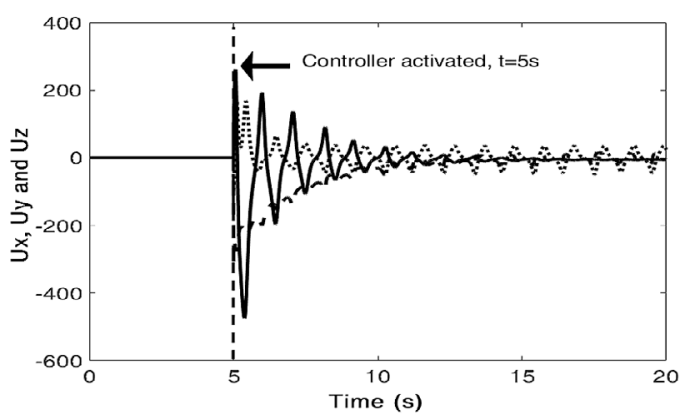

(d)

Figure 7. The switching input $u_{s w x}, u_{s w y}$ and $u_{s w z}$ and $u_{x}, u_{y}$ and $u_{z}$ controller outputs 


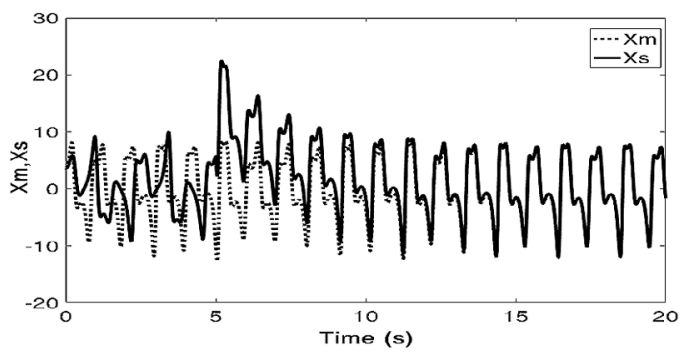

(a)

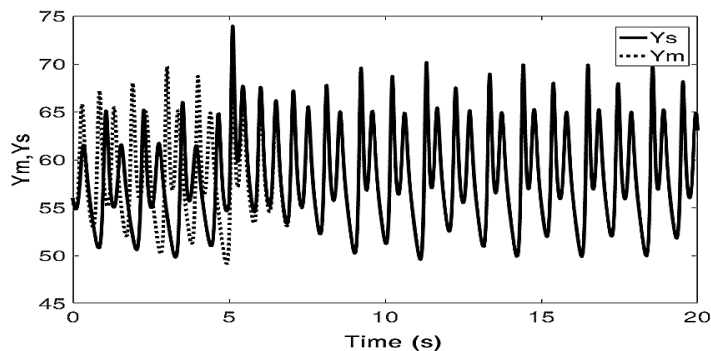

(b)

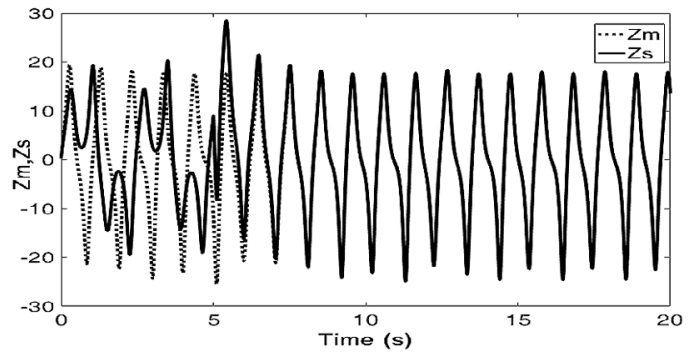

(c)

Figure 8. Simulation results of $x$, $y$ and $z$ variables obtained using SMC method

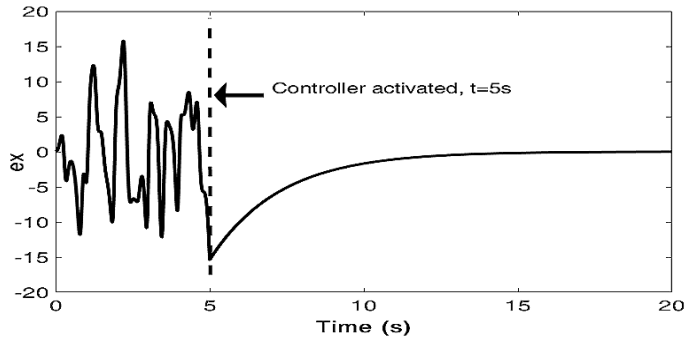

(a)

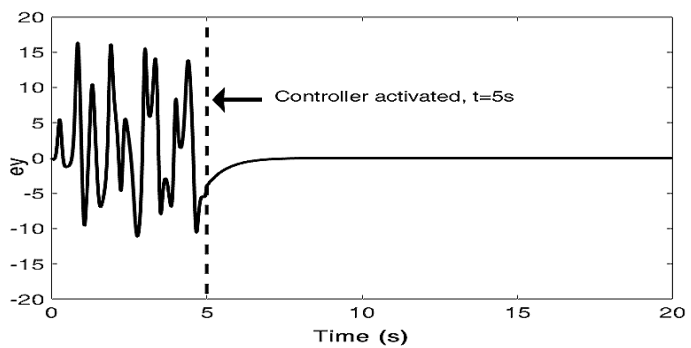

(b)

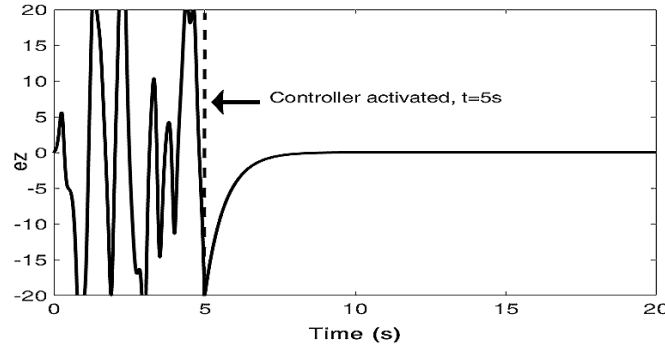

(c)

Figure 9. The $\mathrm{x}, \mathrm{y}$ and $\mathrm{z}$ state variable errors obtained using closed loop system based on SMC system

The PI controller method described in Section 4 was applied to the closed loop control structure based on Fig. 2, and the obtained simulation results are shown in Figures 10 and 11. The adaptive controller method described in Section 5 was applied to the closed loop control structure based on Fig. 4, and the obtained simulation results are illustrated in Figures 12 and 13.

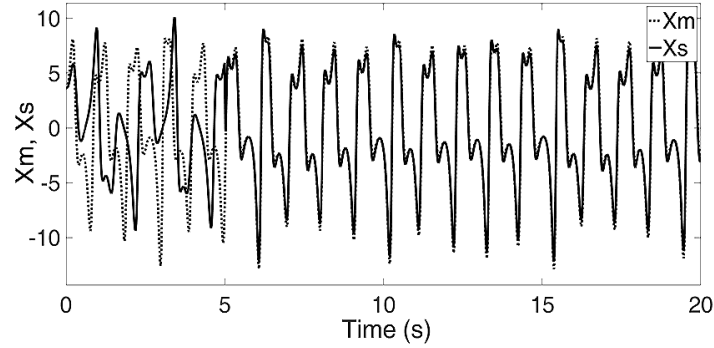

(a)

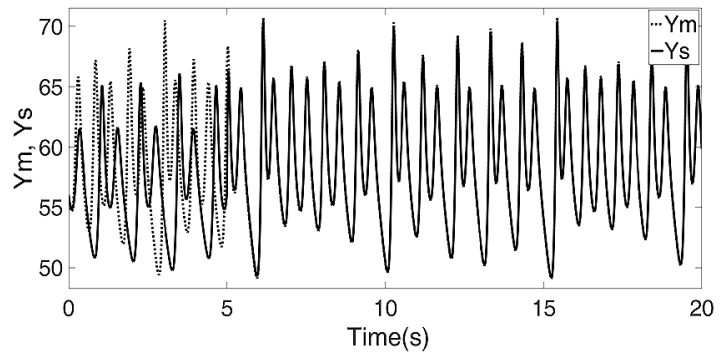

(b)

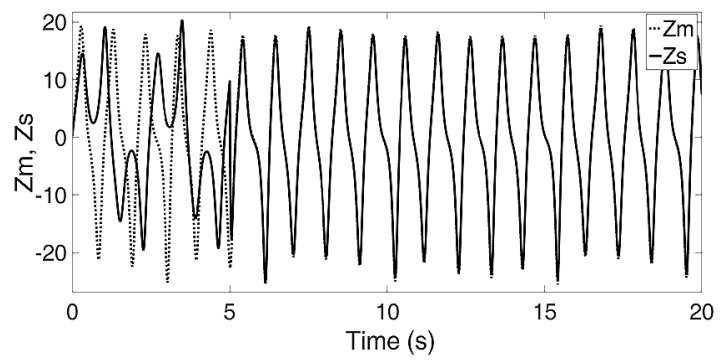

(c)

Figure 10. Simulation results of $\mathrm{x}, \mathrm{y}$ and $\mathrm{z}$ variables obtained using PI control method 


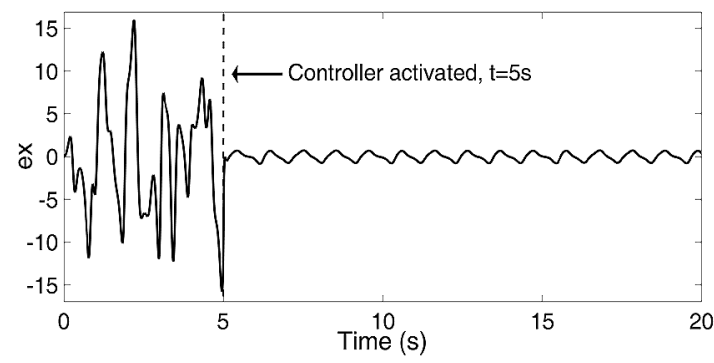

(a)

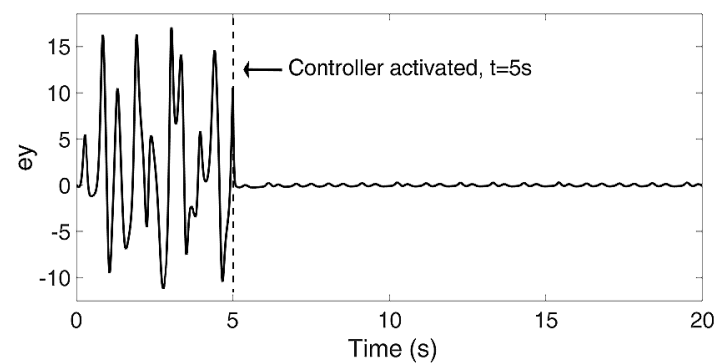

(b)

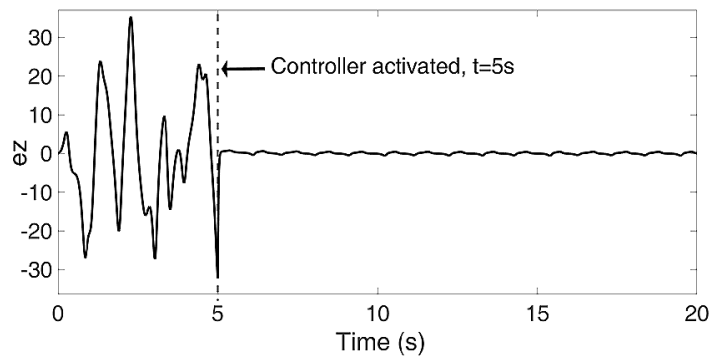

(c)

Figure 11. The $x, y$ and $z$ state variable errors obtained using PI control method

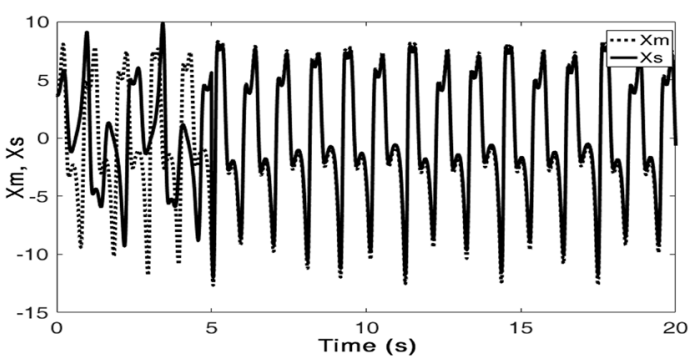

(a)

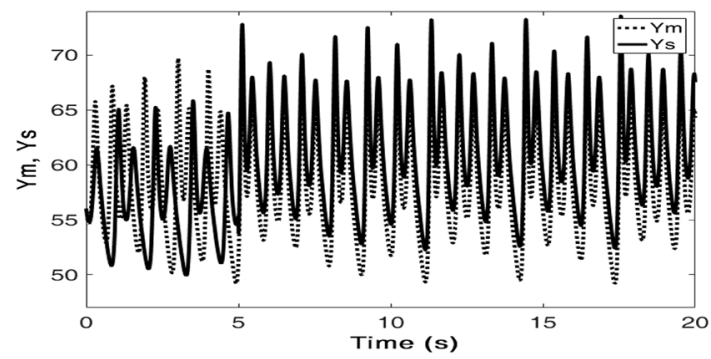

(b)

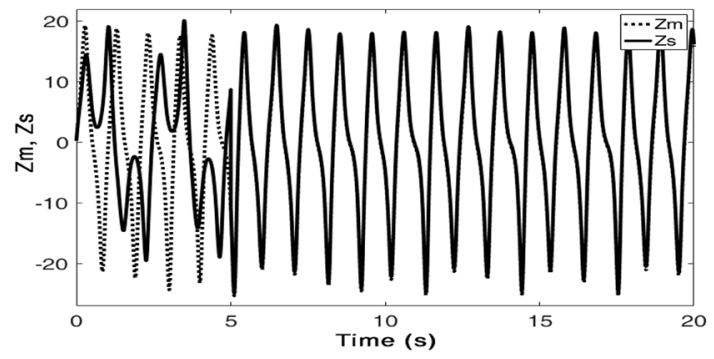

(c)

Figure 12. Simulation results of the $x, y$ and $z$ variables obtained using adaptive control method

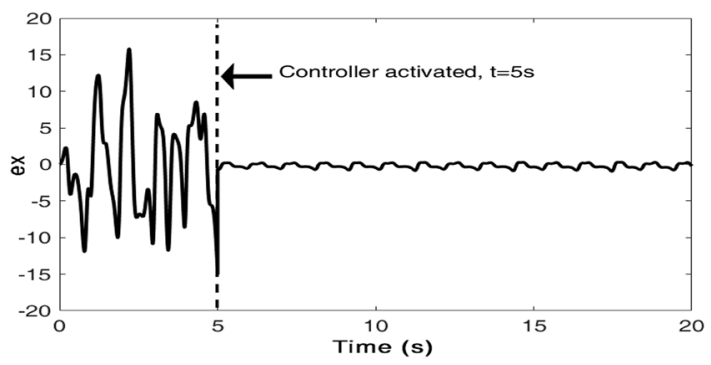

(a)

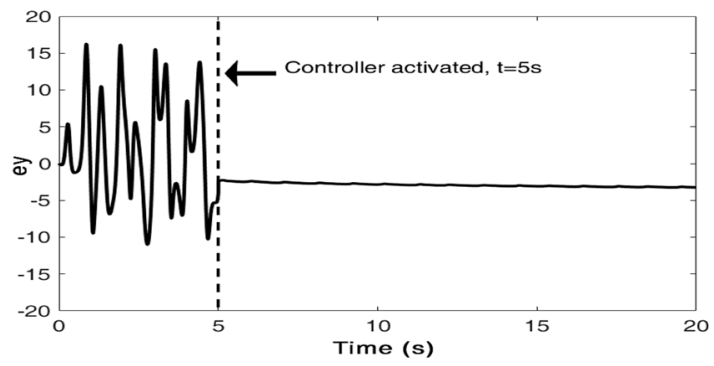

(b)

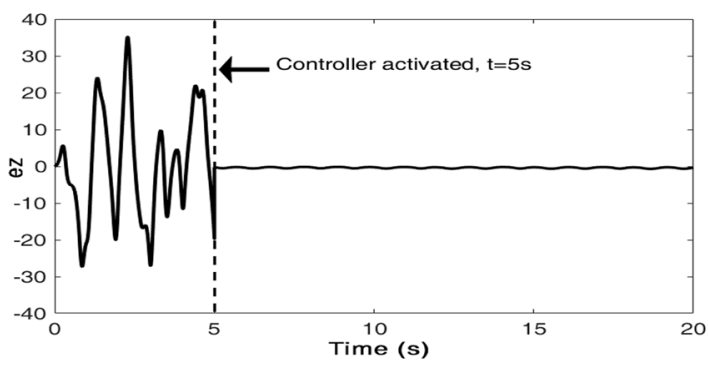

(c)

Figure 13. The $x, y$ and $z$ state variable errors obtained using adaptive control method

The error indice results (Eker, 2012) showing the performances of the controllers are given in Table 2 . The controller was activated in 5 seconds, after then the performance values were obtained in following 18 seconds. In Table 2, sliding mode control method has been better accomplished 
than PI and adaptive control (AdC) methods as the performance index values show.

Table 2. Performance indices of the controllers

\begin{tabular}{|c|c|c|c|c|c|}
\hline \multicolumn{2}{|c|}{} & \multirow{2}{*}{ IAE } & ISE & ITEA & ISCI \\
\hline \multirow{3}{*}{ SMC } & $x$ & 0.0497 & 0.0025 & 0.0224 & 160.5 \\
\cline { 2 - 6 } & $z$ & 0.0128 & 0.0002 & 0.0058 & 22.01 \\
\cline { 2 - 6 } & $z$ & 0.0128 & 0.0002 & 0.0006 & 90.24 \\
\hline \multirow{3}{*}{ PI } & $x$ & 0.4076 & 0.2205 & 0.2329 & 440.3 \\
\cline { 2 - 6 } & $y$ & 0.0911 & 0.0101 & 0.0432 & 28.78 \\
\cline { 2 - 6 } & $z$ & 0.2166 & 0.0660 & 0.1088 & 170.8 \\
\hline \multirow{3}{*}{ AdC } & $x$ & 0.3783 & 0.1870 & 0.1732 & 160.5 \\
\cline { 2 - 6 } & $y$ & 3.1530 & 9.9400 & 1.5700 & 165.1 \\
\cline { 2 - 6 } & $z$ & 0.3999 & 0.1791 & 0.2284 & 1488 \\
\hline
\end{tabular}

The slave state variables can reach the master state variables when the controller activates the system at 5th second as illustrated in Fig. 7, 8, 9, 10, 11, 12 and 13. By using SMC, state variables reached master system behaviour very quickly, whereas using PI and adaptive controls they reached it very slowly. Moreover, when the synchronization errors performance index values of the BLDC motor in Table 2 are examined, sliding mode control response obtains a perfect synchronization in comparison with PI and adaptive control methods.

\section{REFERENCES}

1. Eker, I. (2012). Second-Order Sliding Mode Control with PI Sliding Surface and Experimental Application to an Electromechanical Plant, Arab J Sci Eng., 37, 1969-1986.

2. Hemati, N. \& Leu, M. C. (1992). A complete model characterization of brushless DC motors, IEEE Trans Ind Appl., 28, 172-180.

3. Hou, Y. Y. (2017). Design and implementation of EP-based PID controller for chaos synchronization of Rikitake circuit systems, ISA Transactions, 70, 260-268.

4. Ibrahim, H. E. A., Hassan, F. N. \& Shomer, A. O. (2014). Optimal PID control of a brushless DC motor using PSO and BF techniques, Ain Shams Engineering Journal, 5, 391-398.

5. Karaboga, D. \& Basturk, B. (2007). A powerful and efficient algorithm for

\section{Conclusion}

In this study, three different chaos-based control methods for the brushless DC motor control were proposed. According to the simulation results, Sliding Mode Control showed an effective performance compared with the PI and adaptive controller methods. The results obtained for the three control methods showed that the synchronization method can be used as a good control technique. However, the steady state error values of the adaptive control method were found to be quite high compared to those obtained by PI and SMC methods. Even a very small change in the initial conditions of chaotic systems has lead to significant changes in the dynamic behavior of chaotic systems. According to the resulting $\mathrm{x}, \mathrm{y}$ and $\mathrm{z}$ state variable errors, using SMC based synchronization method, steady-state error, overshoot and settling time have achieved minimum values. It appears that the results obtained from this study could be beneficial in controlling brushless DC motor for electric vehicles and provide different perspectives as this was previously mentioned in studies conducted by Hou (2017) and Rajagopal et al. (2017). The methods which were improved in this study provide a novel approach for chaos-based control of BLDC Motors.

numerical function optimization: artificial bee colony (ABC) algorithm, Journal of Global Optimization, 39, 459-471.

6. Khalil, H. K. (2001). Nonlinear Systems. Prentice Hall, New Jersey, USA.

7. Khan, A., Khattar, B. \& Agrawal, N. (2016). Synchronization of Genesio and Lü chaotic dynamical systems via active control, International Journal of Applied Mathematics, 29,161-174.

8. Köse, E. (2017). Controller design by using non-linear control methods for satellite chaotic system, Electr. Eng., 99, 763-773.

9. Lorenz, E. N. (1963). Deterministic nonperiodic flow, J. Atoms. Sci., 20, 130-141.

10. Pecora, L. M. \& Caroll, T. L. (1990). Synchronization in chaotic systems, Physical Review Letters, 64, 821-825. 
11. Pham, V. T., Kingni, S. T., Volos, C., Jafari, S. \& Kapitaniak, T. (2017). A simple three-dimensional fractional-order chaotic system without equilibrium: Dynamics, circuitry implementation, chaos control and synchronization, $A E U$ - International Journal of Electronics and Communications, $78,220-227$.

12. Premkumar, K. \& Manikandan, B. V. (2015). Speed control of brushless DC motor using bat algorithm optimized adaptive neurofuzzy inference system, Appl Soft Comput., $32,403-419$.

13. Rajagopal, K., Guessas, L., Vaidyanathan, S., Karthikeyan, A. \& Srinivasan, A. (2017). Dynamical analysis and FPGA implementation of a novel hyperchaotic system and its synchronization using adaptive slidingmodecontroland geneticallyoptimized PID control, Mathematical Problems in
Engineering, Article ID 7307452, 14 pages, https://doi.org/10.1155/2017/7307452.

14. Rajagopal, K., Vaidyanathan, S., Karthikeyan, A. \& Duraisamy, P. (2017). Dynamic analysis and chaos suppression in a fractional order brushless DC motor, Electr. Eng., 99, 721-733.

15. Vaidyanathan, S. \& Azar, A. T. (2015). Analysis, Control and Synchronization of a Nine-Term 3-D Novel Chaotic System, Chaos Modeling and Control Systems Design, 19-38.

16. Varshney, A., Gupta, D. \& Dwivedi, B. (2017). Speed response of brushless DC motor using fuzzy PID controller under varying load condition, Journal of Electrical Systems and Information Technology, 4, 310-321. 